Hypotheses

\title{
Natural Substances as New Potential Strategies for the Treatment of Leishmaniosisin Dogs
}

\author{
${ }^{1}$ De Vito Virginia, ${ }^{2}$ Helen Owen, ${ }^{3}$ Amnart Poapolathep and ${ }^{4}$ Giorgi Mario \\ ${ }^{I}$ Department of Veterinary Medicine, University of Sassari, Via Vienna 2, 07100 Sassari, Italy \\ ${ }^{2}$ School of Veterinary Science, University of Queensland, Gatton Campus, Gatton, QLD 4343, Australia \\ ${ }^{3}$ Department of Pharmacology, Faculty of Veterinary Medicine, Kasetsart University, Bangkok 10900, Thailand \\ ${ }^{4}$ Department of Veterinary Sciences, University of Pisa, Via Livornese (lato monte) 1, San Piero a Grado, Pisa, Italy
}

\author{
Article history \\ Received: 10-05-2017 \\ Revised: 04-07-2017 \\ Accepted: 28-08-2017 \\ Corresponding Author: \\ Giorgi Mario \\ Department of Veterinary \\ Sciences, University of Pisa, \\ Via Livornese (lato monte) 1 , \\ San Piero a Grado, Pisa, Italy \\ E-mail: mario.giorgi@unipi.it
}

\begin{abstract}
Leishmaniasis is a disease caused by the protozoan parasites Leishmania, infecting numerous mammal species. Canine leishmaniasis is potentially zoonotic and causes severe fatal disease in dogs. The discovery of new natural products extracted from medicinal plants or compounds derived from them, such as quercetin, hesperidin, vitamin c, horse chestnut extract and selenium could represent a valuable source of new medicinal agents for treating leishmaniasis in dogs.
\end{abstract}

Keywords: Leishmaniasis, Dog, Quercetin, Hesperidin, Vitamin C, Horse Chestnut Extract, Selenium

\section{Introduction}

Leishmaniosisis a disease caused by more than 20 protozoan parasites of the genus Leishmania. These parasites are transmitted by the bite of phlebotomine sand flies and can infect numerous mammalian species, including humans. Leishmaniasis has a distribution ranging through America and Africa and extending into temperate regions of Latin America, Europe and Asia. The World Health Organization has identified human leishmaniasis as one of the most neglected tropical diseases. Visceral leishmaniasis is widespread in more than 80 countries and it is the most serious form, fatal in more than $95 \%$ of cases within 2 years after the onset of the disease. Some $90 \%$ of all new cases are reported from six countries: Brazil, Ethiopia, India, Somalia, South Sudan and Sudan (www.who.int/leishmaniasis/en/).

In contrast, pets are infected with different Leishmania species. L. infantum is the most widespread in domestic animals and dogs represent the main domestic zoonotic reservoir for visceral leishmaniasis. In addition, canine leishmaniasis (CanL) causes severe and often fatal disease in this animal species. Clinical diagnosis in cats of Feline Leishmaniasis syndrome $(\mathrm{FeL})$ in endemic areas is not common. This may be due to their grooming habits that seem to minimize the risk for arthropod infection compared to dogs or the subclinical infection that occurs in most infected cats, or finally, because veterinary practitioners do not usually consider this disease in the list of differential diagnosis of their feline patients (Otranto et al., 2017). Horses and domestic equines suffer occasionally from single or multiple cutaneous lesions but probably they represent an incidental host of the disease (Gramiccia, 2011).

\section{Clinical Signs in Canine Leishmaniasis}

CanL is a severe disease induced by panosomatid species of the genus Leishmania. It is estimated that almost thirty species from the genus Leishmania can be responsible for this disease. L. infantum has been identified as a major, potentially fatal, zoonotic infection in regions of Europe, Africa, Asia and America (Gramiccia and Gradoni, 2005), although other species such as L. chagasi, L. mexicana, L. braziliensis, $L$. donovani or $L$. amazonensis are also potential etiologic agents (Lainson and Shaw, 2005; Alvar et al., 2004). Due to their close association with people, dogs are considered the main reservoir of human infection and phlebotomine sand flies are the biological vectors of this protozoal disease (Reguera et al., 2016; Baneth et al., 2008).

CanL results in several serious clinical signs in infected dogs. The parasite target cell is the macrophage, which becomes the site of parasite replication inhibiting its antimicrobial activities (Gramiccia, 2011). The majority of clinical dogs show poor body condition, generalized muscular atrophy, lymphadenomegaly, excessive skin scaling, decreased appetite and lethargy (Ciaramella et al., 1997; Koutinas et al., 1999; 2001; Baneth et al., 2008). Progressive muscle atrophy is associated with chronic polymyositis characterized by 
the presence of mononuclear infiltrates with Leishmania amastigotes, neutrophilic vasculitis and $\mathrm{IgG}$ immune complexes in muscle tissues in conjunction with serum anti-myofiber antibodies (Vamvakidis et al., 2000). The dermal symptoms include exfoliative, ulcerative, nodular and pustular dermatitis, alopecia and onychogryphosis; in addition, dogs may present with epistaxis, diarrhea and splenomegaly (Noli and Auxilia, 2014; Baneth et al., 2008). Other symptoms are the marked enlargement of lymph nodes caused by the increased number and size of lymphoid follicles and the marked hypertrophy and hyperplasia of medullary macrophages in the cords and sinuses (Lima et al., 2004). The splenomegaly is associated with increased monocyte and macrophage cellularity and changes in the microvasculature structure with abundant pulp venules and veins and increased reticular fibers (Alexandre-Pires et al., 2006). Renal disease can be the result of tubulointerstitial nephritis, glomerulonephritis and amyloidosis with a progression from asymptomatic proteinuria to nephrotic syndrome or chronic renal disease with azotemia (Koutinas et al., 1999). Joint and bone lesions are frequently present with periosteal and intra-medullary proliferative lesions and cortical and medullary osteolysis (Nieto et al., 1992; Agut, et al., 2003; Blavier et al., 2001). Ocular lesions are present in 16 to $80.5 \%$ of dogs and consist of anterior uveitis, conjunctivitis, dry keratoconjunctivitis and blepharitis (Ciaramella et al., 1997; Koutinas et al., 1999; 2001; Naranjo et al., 2005; Peña et al., 2000). Epistaxis, hematuria and hemorrhagic diarrhoea are associated with tissue ulceration and alterations in primary and secondary hemostasis. Hemostatic disorders include platelet aggregation abnormalities leading to platelet dysfunction, thrombocytopenia and decreased coagulation factor activities (Ciaramella et al., 2005). Profuse epistaxis can be the only presenting sign of disease and can cause death because of uncontrollable blood loss secondary to pyogranulomatous or lymphoplasmacytic rhinitis, thrombocytopathy and hyperglobulinemia (Juttner et al., 2001). Anemia is present in the majority of symptomatic dogs because of chronic renal disease or decreased erythropoiesis caused by chronic disease and can be aggravated by blood loss or immune mediated destruction of red blood cells (Ciaramella et al., 1997; Koutinas et al., 1999; 2001).

\section{Current Therapies for the Treatment of CanL}

Several drugs used for the treatment of CanL are able to improve clinical signs temporarily or cure dogs clinically, but none of these treatments reliably eliminates the infection (Miró et al., 2008). Nowadays, chemotherapy is the most efficient treatment for sick dogs and shows benefits in terms of reduction of prevalence and incidence of the disease, as well as in the control of pathogen's life cycle (Miró et al., 2011).
Current chemotherapy is based on monotherapy or combination therapy of leishmanicidal and leishmaniostatic drugs. Disadvantages of these firstchoice drugs are that they require parenteral delivery by veterinarian practitioners and the treatment is long and unpleasant for dogs and owners. Undesirable toxic side effects, particularly initiation or exacerbation of renal disease are not uncommon (Miró et al., 2008; Valladares et al., 1998). Furthermore, relapses are extremely frequent (up to $74 \%$ ) in the first year posttreatment (Slappendel and Teske, 1997). Finally, development of drug-resistant strains of the parasite can be a consequence of drug misuse or can be due to host factors (modifications of pharmacokinetic parameters or an alteration in the immunological response to the parasite) and parasite factors (structural modifications of target proteins or mechanisms to evade the host immunological system) (Rhalem et al., 1999).

\section{Prevention (or Preventive Measures)}

Vaccines are an alternative to chemotherapy drugs. These stimulate the immune response with the aim of preventing infection and disease progression and blocking the parasite's life cycle. Many studies in animal models have shown that after inoculation of infective metacyclic promastigotes by the sand fly, skin dendritic cells are responsible for phagocytosis and antigen presentation to $\mathrm{T}$ cells. Afterwards, they migrate to the nearest lymph node to present the antigens to immature $\mathrm{T}$ cells and cause proliferation of CD4+ and CD8+ $\mathrm{T}$ cells. At this moment, a classical dichotomous cell-mediated response is triggered, this can be protective (Th1) or not protective (Th2), thus resulting in resistance or susceptibility to Leishmania infection. Vaccines can adequately boost the immunological system against a potential infection, thus avoiding the toxicity of chemotherapy and the emergence of resistant strains. However, prevention of CanL also involves reducing exposure to vectors using formulations with repellent/insecticide activity such us a large number of insecticide containing deltamethrin or combination of permethrin and neonicotinoid insecticide (Reguera et al., 2016).

\section{New Alternatives from Natural Products}

Extracts from medicinal plants or compounds derived from them are a valuable source of new medicinal agents for the treatment of leishmaniasis. There is a large range of families and species of plants with a potentially active leishmanicidal effect. The leishmanicidal effect may reside in phytochemical components such as flavonoids and, specifically, quercetin, which is a strong candidate in the combination therapy for the treatment of (Marìn et al., 2009) 


\section{Quercetin}

Quercetin is a naturally occurring flavonoid isolated from Fagopyrum esculentum (Polygonaceae) (Polonio and Efferth, 2008). This compound shows potent anti-Leishmania activity in vitro on the amastigote stage of $L$. donovani and $L$. amazonensis. Most of the beneficial health effects of this flavonoid are attributed to its antioxidant and chelating activities, via scavenging of reactive oxygen species/metal chelation and stimulation/inhibition of enzyme activities/signal transduction pathways. Concerning its mechanism of action, quercetin is able to induce topoisomerase IImediated kinetoplastid DNA minicircle cleavage in $L$. donovani promastigotes and intracellular amastigotes. The treatment of promastigotes with quercetin leads to cell cycle arrest in the G0/G1 phase followed by apoptotic cell death. Quercetin is a specific inhibitor of topoisomerase I, which is an unusual bi-subunit topoisomerase in Leishmania. Additionally, this compound can chelate iron, which translates into a decreased availability of the iron dependent ribonucleotide reductase, a rate limiting enzyme for DNA synthesis (Polonio and Efferth, 2008; Sen and Chatterjee, 2011; Sen et al., 2005; Fiorania et al., 2010; Sen et al.,2008; Da Silva et al., 2012).

In recent years, quercetin is becoming increasingly popular as a dietary supplement for pets. This compound has been suggested to have various beneficial effects like preventing cancer, suppressing inflammation or decreasing fat absorption and agerelated diseases that are associated with increased oxidative stress (Reinboth et al., (2010).

In a study by Reinboth et al. (2010), a single administration of quercetin in dogs at the dose of 150 $\mathrm{mg} /$ subject (about $10 \mathrm{mg} \mathrm{kg}^{-1}$ ) showed a maximum plasma concentration $\left(\mathrm{C}_{\max }\right)$ of $234 \mathrm{nmol} \mathrm{L}^{-1}$ (equivalent to $0.77 \mu \mathrm{g} \mathrm{mL}^{-1}$ ) and a very low bioavailability (4\%). These results are in agreement with different studies present in literature on other animal species. Indeed, in rats and pigs the absolute bioavailability of quercetin was only 5 and 1\%, respectively (Wessmann et al., 2000; Chen et al., 2005). Due to the low bioavailability of quercetin after oral administration in different animal species, a study performed by Medalpharma sas industry in 2012 (internal data) investigated the pharmacokinetics of quercetin in dogs after a single administration at $150 \mathrm{mg} \mathrm{kg}^{-1}$ b.w. No visible side effects were observed immediately after the single administration or over the subsequent seven days. The $\mathrm{C}_{\max }$ of quercetin after a single dose (150 mg kg-1 b.w.) was $11 \mu \mathrm{g} \mathrm{mL}^{-1}$. Findings of this study suggest that, if compared with a previous study in dogs (Reinboth et al., 2010; dose: $10 \mathrm{mg} \mathrm{kg}^{-1}$; $\mathrm{C}_{\max }: 0.77 \mu \mathrm{g} \mathrm{mL}^{-1}$ ), quercetin is expected to have linear first-order kinetics in dogs for doses ranging from 10 to $150 \mathrm{mg} \mathrm{kg}^{-1}$. Unfortunately, no pharmacokinetic/pharmacodynamic studies are available concerning a clinical dose of quercetin for the treatment of CanL.

The IC 50 (50\% inhibition concentration of cell growth) value against $L$. donovani and $L$. amazonensis intracellular amastigotes is $1.0 \mu \mathrm{g} \mathrm{mL}^{-1}$. If this value is multiplied for $24 \mathrm{~h}$, a value of $\mathrm{AUC}_{0-24 \mathrm{~h}}$ of $24 \mu \mathrm{g}^{*} \mathrm{~h} \mathrm{~mL} \mathrm{~m}^{-1}$ is calculated. This parameter represents the theoretical value to maintain the plasma concentration of quercetin above the IC 50 for $24 \mathrm{~h}$. Bearing in mind the dose proportional feature of quercetin and tha ta dose of 150 $\mathrm{mg} \mathrm{kg}{ }^{-1}$ showed a value of $\mathrm{AUC}_{0-24 \mathrm{~h}}: 129 \mu \mathrm{g}^{*} \mathrm{~h} \mathrm{~mL}$ (Medalpharma sas, internal data, 2012), it can be calculated that a dose of $28 \mathrm{mg} \mathrm{kg}^{-1}$ produces an $\mathrm{AUC}_{0 \text { - }}$ ${ }_{24 \mathrm{~h}} 24 \mu \mathrm{g}^{*} \mathrm{~h} \mathrm{~mL}{ }^{-1}$ maintaining the plasma concentration of quercetin above the IC 50 for one day.

\section{Hesperidin}

Hesperidin is an abundant flavonoid produced by citrus cultivations and it is the major product of oranges and lemons. It is usually administered in association with vitamin $\mathrm{C}$ to increase the efficacy of the compound and it has been shown to possess an excellent safety profile (Garg et al., 2001).

Due to its broad spectrum of activities, hesperidin is effectively used as a supplemental agent in numerous treatment programmes. One of the main effect of hesperidin is on the vascular system. Hesperidin supplementation has been used in patients suffering with blood vessel disorders including those involving abnormal permeability and fragility of capillary walls (resulting in oedema, bleeding and hypertension) (Garg et al., 2001). The role of hesperidin in increasing capillary resistance has been attributed to its inhibitory effect on the action of hyaluronidase enzyme that accentuate capillary permeability and fragility. In a study on human patients, hesperidin inhibited epinephrine and ADP-induced platelet aggregation at a concentration of $0.08 \mathrm{mg} \mathrm{mL}^{-1}$. In another study on horses, hesperidin was found to effectively reduce aggregation of erythrocytes. This decrease in erythrocyte aggregation may explain the beneficial effects of hesperidin on the abnormal permeability and fragility of capillary walls. Hesperidin has been shown to possess significant anti-inflammatory and analgesic effects. This effect could be attributed to the inhibition of the release of histamine from basophils by hesperidin or its metabolic products. This compound showed anti-infective and anti-replicative activities in vitro against several plant and animal microbes (Garg et al., 2001). Finally, hesperidin has been reported to possess antioxidant and immuno-suppressant properties. Indeed, after intragastric administration of hesperidin at the dose of $50 \mathrm{mg} \mathrm{kg}$, this compound suppressed bacterial alphaamylase antibody production in mice and significantly increased the development of immunological memory in the cellular immune response (Kim and Cho, 1991). 
Concerning the pharmacokinetics of hesperidin, in a study by Ameer et al. (1996), the authors evaluated the oral absorption of hesperidin in healthy young human volunteers at the dose of $500 \mathrm{mg} /$ subject (about $7 \mathrm{mg}$ $\mathrm{kg}^{-1}$ ) showing a low bioavailability (about 25\%). Furthermore, a study performed by Medalpharma sas industry in 2012 (internal data), reported the pharmacokinetics of hesperidin in dogs after a single administration at the dose of $150 \mathrm{mg} \mathrm{kg}^{-1}$ b.w. No visible side effects were observed immediately after the single administration or over the subsequent seven days. The $\mathrm{C}_{\max }$ of hesperidin after a single dose (150 $\mathrm{mg} \mathrm{kg}^{-1}$ b.w.) was $7 \mu \mathrm{g} \mathrm{mL}^{-1}$.

No pharmacokinetic/pharmacodynamic studies are available concerning the clinical dose of hesperidin for the treatment of CanL. From the studies reported above it can be summarized that doses from 7 to 50 $\mathrm{mg} \mathrm{kg}^{-1}$ might produce suitable plasma concentrations of hesperidin to effectively treat CanL, while higher doses up to $150 \mathrm{mg} \mathrm{kg}^{-1}$ were shown not to evoke appreciable adverse effects.

\section{Vitamin $C$}

Leishmaniosis is accompanied by severe anemia and shortened lifespan of erythrocytes. Several lines of evidence suggest an important role of enhanced lipid peroxidation in the pathogenesis of hemolytic anemia. In healthy animals the cells invest much of their metabolic activity in the reductive processes that combat the threat of oxidation. Glutathione (GSH), with glutathione redox cycle in the cell, is involved in several defence processes against oxidative damage. Obviously, a deficiency of these antioxidants increases cells' susceptibility to oxidative damage.

Vitamin C (ascorbic acid) is a powerful antioxidant that reacts rapidly with a variety of oxidants, including the rather poorly reactive superoxide anion radical (Bildik et al., 2004). It is transported into erythrocytes as dehydroascorbic acid, which is then reduced to ascorbate via a GSH-dependent reaction.

A study by Field and Rekers, (1949) showed that supplementary treatment with vitamin C in dogs (100 $\mathrm{mg}$ orally three times daily for 1 week) might potentiate the anti-hemorrhagic action of the flavanones quercetin. Furthermore, vitamin $\mathrm{C}$ is usually administered in association with another flavonoid hesperidin to increase the efficacy of the final compound, this combination has been shown to be extremely safe and without side effects (Garg et al., 2001).

In light of the features mentioned above, vitamin $\mathrm{C}$ might be a potential new natural compound for the treatment some clinical signs of CanL.

\section{Horse Chestnut Extract}

The active compound $\beta$-aescin is the main saponin isolated from the seeds of the horse-chestnut Aesculus hippocastanumi (Sapindaceae). A study of Vermelho et al. (2014) showed a $\beta$-aesc in value equal to $1.04 \pm 0.23 \mathrm{ug}$ $\mathrm{mL}^{-1}$ for $L$. infantum amastigotes and the Horse Chestnut Extract (HCE) contains 70\% esc (Guillaume and Padioleau, 1994).

Concerning its mechanism of action, HCE showed antiradical activity in both in vitro and in vivo studies inhibiting both enzymatic and non-enzymatic in vitro lipid peroxidation (in the concentration range $5 \times 10^{-6}$ to $5 \times 10^{-4} \mathrm{~g} \mathrm{~mL}^{-1}$ ). Furthermore, additional studies have shown the veinotonic and lymphagogue properties of HCE at different dosages. Indeed, HCE dose dependently contracts the canine saphenous isolated vein for more than $5 \mathrm{~h}$. In the perfused canine saphenous vein, HCE (25-50 mg in bolus) increases the venous pressure of the normal vein and the pathological vein by increasing the contractile response to noradrenaline. During the perfusion in the inverse direction of the blood flow, a clear contracting effect on the valves is also noted with HCE. In the anaesthetized dog, HCE in situ improves the femoral vein compliance and opposes the venous distension obtained during clamping in a carotido-femoral perfusion with constant flow. HCE significantly increases femoral venous pressure and flow, together with thoracic lymphatic flow, while respecting the arterial parameters ( 2.5 and $5 \mathrm{mg} \mathrm{kg}^{-1}$ intravenously). In regards to the vasculotropic action, HCE dose dependently diminishes the cutaneous capillary hyper permeability induced either by injections of inflammatory agents such as histamine and serotonin in the rat (100 to $400 \mathrm{mg} \mathrm{kg}^{-1}$ orally), or by an irritating agent like chloroform applied in the rabbit (50 to $300 \mathrm{mg}$ $\mathrm{kg}^{-1}$ orally and 2.5 to $5 \mathrm{mg} \mathrm{kg}^{-1}$ intravenously). It significantly increases the vascular resistance in guinea pigs fed a scorbutigenic diet as measured by the petechia method (50 to $400 \mathrm{mg} \mathrm{kg}^{-1}$ orally). Regarding antiedema and anti-inflammatory activities, HCE decreases the formation of edema induced in the rat's hind paw, one of lymphatic origin, the other of inflammatory origin (200 to $400 \mathrm{mg} \mathrm{kg}^{-1}$ orally). In an experimental model of pleurisy in rats, HCE suppresses plasmatic extravasation and leucocytes emigration into the pleural cavity (200 to $400 \mathrm{mg} \mathrm{kg}{ }^{-1}$ oraly; 1 to $10 \mathrm{mg} \mathrm{kg}^{-1}$ intravenously). Finally, HCE decreases connective tissue formation in the subchronic, inflammatory granuloma model in the rat (400 $\mathrm{mg} \mathrm{kg}^{-1}$ orally and $5-10 \mathrm{mg} \mathrm{kg}^{-1}$ subcutaneously) (Guillaume and Padioleau, 1994).

Due to its broad mechanism of action, HCE might be considered a suitable supplementary therapy for the treatment of the main clinical signs of CanL.

\section{Selenium}

The final compound considered in the present work is selenium, a micronutrient element with broad functions in biological systems. Selenium ions $\left(\mathrm{SeO}_{2}\right.$ is the chemical form of selenium) have antioxidant, cancer preventing and antiviral activities and also appear to 
improve the immune response of hosts against various species of bacteria and viral antigens (Beheshti et al., 2013; Rayman, 2012; Soflaei et al., 2014; Tapiero et al., 2003). The $\mathrm{IC}_{50}$ of selenium for $L$. infantum is $50 \mu \mathrm{g}$ $\mathrm{mL}^{-1}$ (Soflaei et al., 2014).

Considering selenium activities and its $\mathrm{IC}_{50}$ value, this compound might be used against leishmaniasis with dose-dependent anti-leishmanial activities.

\section{Conclusion}

Leishmaniosisis a neglected, potentially lethal infectious disease for human and pets caused by parasites of the genus Leishmania that affects many developing countries.

The treatment of leishmaniosis in pets is challenging and lengthy. Therapeutic problems include the toxicity of available drugs and increasing drug resistance. Leishmaniosis has received scarce attention from governments and the pharmaceutical industries until the past decade.

The development of new parasite targets and synthetic drugs along with research on natural products such as quercetin, hesperidin, vitamin $\mathrm{C}$, horse chestnut extract and selenium might represent a possible strategy for the discovery of new therapeutic agents against Leishmania. Although further studies are required to determine if these natural products can be used in the same formulation, these preliminary findings pave the road for their use as a supportive treatment for leishmaniosis in dogs.

\section{Acknowledgment}

None of the authors have any financial or personal relationships that could inappropriately influence or bias the content of the paper.

\section{Funding Information}

The preparation of manuscript was not supported by any external funding.

\section{Author's Contributions}

De Vito Virginia and Giorgi Mario: Contributed to conception of the study, analysis of the literature and wrote the manuscript.

Helen Owen and Amnart Poapolathep: Contributed to the analysis of the literature and edited the manuscript.

\section{References}

Agut, A., N. Corzo, J. Murciano, F.G. Laredo and M. Soler, 2003. Clinical and radiographic study of bone and joint lesions in 26 dogs with leishmaniasis. Vet. Rec., 153: 648-652.
Alexandre-Pires, G., D. Pais, M. Correia and J.A. Pina, 2006. Leishmaniosis-a report about the microvascular and cellular architecture of the infected spleen in Canis familiaris. Microsc. Res. Tech., 69: 227-235

Alvar, J., C. Canavate, R. Molina, J. Moreno and J. Nieto, 2004. Canine leishmaniasis. Adv. Parasitol., 57: 1-88.

Ameer, B., R.A. Weintraub, J.V. Johnson, R.A.Yost and R.L.Rouseff, 1996. Flavanone absorption after naringin, hesperidin and citrus administration. Clin. Pharmacol. Ther., 60: 34-40.

Baneth, G., A.F. Koutinas, L. Solano-Gallego, P. Bourdeau and L. Ferrer, 2008. Canine leishmaniosis-new concepts and insights on an expanding zoonosis: Part one. Trends Parasitol., 24: 324-330.

Beheshti, N., S. Soflaei, M. Shakibaie, M.H. Yazdi and F. Ghaffarifar et al., 2013. Efficacy of biogenic selenium nanoparticles against Leishmania major: In vitro andin vivo studies. J. Trace Elem. Med. Bio., 27: 203-207.

Bildik, A., F. Kargın, K. Seyrek, S. Pasa and S. Ozensoy, 2004. Oxidative stress and non-enzymatic antioxidative status in dogs with visceral Leishmaniasis. Res. Vet. Sci., 77: 63-66.

Blavier, A., S. Keroack, P. Denerolle, I. Goy-Thollot and L. Chabanne et al., 2001. Atypical forms of canine leishmaniasis. Vet. J., 162: 108-120.

Chen, X., O.Q. Yin, Z. Zuo and M.S.S. Chow, 2005. Pharmacokinetics and modeling of quercetin and metabolites. Pharm. Res., 22: 892-901.

Ciaramella, P., A. Pelagalli, L. Cortese, M.E. Pero and M. Corona et al., 2005. Altered platelet aggregation and coagulation disorders related to clinical findings in 30 dogs naturally infected by Leishmania infantum. Vet. J., 169: 465-467.

Ciaramella, P., G. Oliva, R.D. Luna, L. Gradoni and R. Ambrosio et al., 1997. A retrospective clinical study of canine leishmaniasis in 150 dogs naturally infected by Leishmania infantum. Vet. Rec., 141: 539-543.

Da Silva, E.R., C.C. Maquiaveli and P.P. Magalhães, 2012. The leishmanicidal flavonols quercetin and quercitrin target Leishmania (Leishmania) amazonensis arginase. Exp. Parasitol., 130: 183-188.

Field, J.B. and E.P. Rekers, 1949. Studies of the effects of flavonoids on roentgen irradiation disease. Part II, comparison of the protective influence of some flavonoids and vitamin $\mathrm{C}$ in dogs. J. Clin. Invest., 28: 746-751.

Fiorania, M., A. Guidarelli, M. Blasa, C. Azzolini and M. Candiracci et al., 2010. Mitochondria accumulate large amounts of quercetin: Prevention of mitochondrial damage and release upon oxidation of the extramitochondrial fraction of the flavonoid. J. Nutr. Biochem., 21: 397-404. 
Garg, A., S. Garg, L.J.D. Zaneveld and A.K. Singla, 2001. Chemistry and pharmacology of the citrus bioflavonoid hesperidin. Phytother. Res., 15: 655-669.

Gramiccia, M., 2011. Recent advances in leishmaniosis in pet animals: Epidemiology, diagnostics and antivectorial prophylaxis. Vet. Parasitol., 181: 23-30.

Gramiccia, M. and L. Gradoni, 2005. The current status of zoonotic leishmaniases andapproaches to disease control. Int. J. Parasitol., 35: 1169-1180.

Guillaume, M. and F. Padioleau, 1994. Veinotonic effect, vascular protection, antiinflammatory and free radical scavenging properties of horse chestnut extract. Arzneimittel-Forschung, 44: 25-35.

Juttner, C., M. Rodríguez Sánchez, E. Rollán Landeras, R.J. Slappendel and C. Fragío Arnold, 2001. Evaluation of the potential causes of epistaxis in dogs with natural visceral leishmaniasis. Vet. Rec., 149: 176-179.

Kim, C.J. and S.K. Cho, 1991. Pharmacological activities of flavonoids (II)-structure activity relationships of flavonoids in immunosuppression. Arch. Pharmacol. Res., 14: 147-159.

Koutinas, A.F., M.N. Saridomichelakis, M.E. Mylonakis, L. Leontides and Z. Polizopoulou et al., 2001. A randomised, blinded, placebo-controlled clinical trial with allopurinol in canine leishmaniasis. Vet. Parasitol., 98: 247-261.

Koutinas, A.F., Z.S. Polizopoulou, M.N. Saridomichelakis, D. Argyriadis and A. Fytianou et al., 1999. Clinical considerations on canine visceral leishmaniasis in Greece: A retrospective study of 158 cases (19891996). J. Am. Anim. Hosp. Assoc., 35: 376-383.

Lainson, R. and J.J. Shaw, 2005. Leishmaniasis in the new world. In: Topley and Wilson's Microbiology and MicrobialInfections, Collier, L., A. Balows and M. Sussman (Eds.), Parasitology, Arnold, London, pp: 313-349.

Levine, M., C. Conry-Cantilena, Y. Wang, R.W. Welch and P.W. Washko et al., 1996. Vitamin C pharmacokinetics in healthy volunteers: Evidence for a recommended dietary allowance (ascorbic acid/bioavailability). Proc. Natl. Acad. Sci. U S A, 93: 3704-3709.

Lima, W.G., M.S. Michalick, M.N. De Melo and W.L. Tafuri, 2004. Canine visceral leishmaniasis: A histopathological study of lymph nodes. Acta Trop., 92: 43-53.

Marìn, C., S. Boutaleb-Charki, J.G. Diaz, O. Huertas and M.J. Rosales et al., 2009. Antileishmaniasis activity of flavonoids from consolida oliweriana. J. Nat. Prod., 72: 1069:1074.

Miró, G., L. Cardoso, M.G. Pennisi, G. Oliva and G. Baneth, 2008. Canine leishmaniasis-new concepts and insights on an expanding zoonosis: Part two. Trends Parasitol., 24: 371-377.
Miró, G., R. Gálvez, C. Fraile, M.A. Descalzo and R. Molina, 2011. Infectivity to Phlebotomus perniciosus of dogs naturally parasitized with Leishmania infantum after different treatments. Parasit. Vectors., 4: 52-52.

Naranjo, C., D. Fondevila, M. Leiva, X. Roura and T. Peña, 2005. Characterization of lacrimal gland lesions and possible pathogenic mechanisms of keratoconjunctivitis sicca in dogs with leishmaniosis. Vet. Parasitol. 133: 37-47.

Nieto, C.G., I. Navarrete, M.A. Habela, F. Serrano and E. Redondo, 1992. Pathological changes in kidneys of dogs with natural Leishmania infection. Vet. Parasitol., 45: 33-47.

Noli, C. and S.T. Auxilia, 2005. Treatment of canine Old World visceral leishmaniasis: Asystematic review. Vet. Dermatol., 16: 213-232.

Otranto, D., E. Napoli, M.S. Latrofa, G. Annoscia and V.D Tarallo et al., 2017. Feline and canine leishmaniosis and other vector-borne diseases in the Aeolian Islands: Pathogen and vector circulation in a confined environment. Vet. Parasitol., 236: 144-151.

Peña, M.T., X. Roura and M.G. Davidson, 2000. Ocular and periocular manifestations of leishmaniasis in dogs: 105 cases (1993-1998). Vet. Ophthalmol., 3: 35-41.

Polonio, T. and T. Efferth, 2008. Leishmaniasis: Drug resistance and natural products (Review). J. Mol. Med., 22: 277-286.

Rayman, M.P., 2012. Selenium and human health. Lancet., 379: 1256-1268.

Reguera, R.M., M. Morán, Y. Pérez-Pertejo, C. GarcíaEstrada and R. Balana-Foucea, 2016. Current status on prevention and treatment of canine leishmaniasis. Vet. Parasitol., 227: 98-114.

Reinboth, M., S. Wolffram, G. Abraham, F.R. Ungemach and R. Cermak, 2010. Oral bioavailability of quercetin from different quercetin glycosides in dogs. Br. J. Nutr., 104: 198-203.

Rhalem, A., H. Sahibi, S. Lasri amd C.L. Jaffe, 1999. Analysis of immune responses in dogs with canine visceral leishmaniasis before and after, drug treatment. Vet. Immunol. Immunopathol. 71: 69-76.

Sen, G., S. Mukhopadhyay, M. Ray and T. Biswas, 2008. Quercetin interferes with iron metabolism in Leishmania donovani and targets ribonucleotide reductase to exert leishmanicidal activity. J. Antimicrob. Chemother., 61: 1066-1075.

Sen, R. and M. Chatterjee, 2011. Plant derived therapeutics for the treatment of Leishmaniasis. Phytomedicine, 18: 1056-1069.

Sen, G., S. Mandal, S.S. Roy, S. Mukhopadhyay and T. Biswas, 2005. Therapeutic use of quercetin in the control of infection and anemia associated with visceral leishmaniasis. Free Radic. Biol. Med., 38: 1257-1264. 
Slappendel, R.J. and E. Teske, 1997. The effect of intravenous or subcutaneousadministration of meglumine antimonate (Glucantime) in dogs withleishmaniasis. A randomized clinical trial. Vet. Q., 19: 10-13.

Soflaei, S., A. Dalimi, A. Abdoli, M. Kamali and V. Nasiri et al., 2014. Anti-leishmanial activities of selenium nanoparticles and selenium dioxide on Leishmania infantum. Comp. Clin. Pathol., 23: 15-20.

Tapiero, H., D.M. Townsend and K.D. Tew, 2003. The antioxidant role of selenium and selenocompounds. Biomed. Pharmacother., 57: 134-144. DOI: $10.1016 / \mathrm{S} 0753-3322(03) 00035-0$

Valladares, J.E., C. Riera, J. Alberola, M. Gállego and M. Portús et al., 1998. Pharmacokinetics of meglumine antimoniate after administration of a multiple dose in dogs experimentally infected with Leishmania infantum. Vet. Parasitol., 75: 33-40. DOI: 10.1016/S0304-4017(97)00193-3
Vamvakidis, C.D., A.F. Koutinas, G. Kanakoudis, G. Georgiadis and M. Saridomichelakis, 2000. Masticatory and skeletal muscle myositis in canine leishmaniasis (Leishmania infantum). Vet. Rec., 146: 698-703.

Vermelho, B.A., C.T. Supuran, V. Cardoso, D. Menezes and J.R. De Andrade Silva et al., 2014. Leishmaniasis: Possible new strategies for treatment. In: Leishmaniasis-Trends in Epidemiology, Diagnosis and Treatment. Claborn, D.M.

www.who.int/leishmaniasis/en/

Wessmann, A.P. and S. Wolffram, 2000. Bioavailability and metabolism of the flavonol quercetin in the pig. Free Radic. Biol. Med., 28: 1056-1067. 